\title{
Chronic microsporidian infection of the nasal mucosae, sinuses and conjunctivae in HIV disease
}

\author{
C J N Lacey, A M T Clarke, P Fraser, T Metcalfe, G Bonsor, A Curry
}

\begin{abstract}
A case of chronic infection of the nasal mucosae, sinuses and conjunctivae with a microsporidian parasite in association with HIV infection and immune deficiency is reported. This microsporidian resembles both Encephalitozoon cuniculi and the newly described Encephalitozoon hellem by electron microscopy. This occurred in an adult male resident in the $U K$ with no history of foreign travel. Although there are previous descriptions of conjunctival infections from the USA, this is the first description of infection of the nasal epithelium. Further studies are underway to classify this protozoan.
\end{abstract}

\section{Introduction}

Microsporidia are obligate, intacellular, spore-forming protozoal parasites. They are widespread in the natural world and have long been recognised as a cause of disease in nonhuman hosts. However, only six microsporidial infections in patients without HIV infection have been documented. ${ }^{1}$ Reports of microsporidial infection in patients with AIDS first appeared in $1985,,^{23}$ describing a new genus and species Enterocytozoon bienusi, predominantly infecting the small intestine and usually presenting with chronic diarrhoea and weight loss. Studies have suggested that $30 \%$ of patients with HIV disease and pathogen negative chronic diarrhoea have Enterocytozoon bienusi infection. ${ }^{45}$ Recent work utilising duodenal biopsy specimens from HIV infected individuals with and without diarrhoea, and presumptively HIV negative individuals has provided evidence of the pathogenicity of Enterocytozoon bienusi, without evidence of a state of commensal carriage. ${ }^{6}$

Encephalitozoon cuniculi usually infects non-human mammals. Infection usually begins in intestinal epithelial cells, and in animals first liver and then other extra-intestinal sites are infected, presumably via blood, lymph, or infected macrophages. ${ }^{7}$ In late infection central nervous system vasculitis and interstitial nephritis predominate. Two nonHIV infected children with encephalitozoon infection and CNS involvement have been described. ${ }^{1}$ Infection with protozoa morphologically identical to Encephalitozoon cuniculi was first described in HIV infection in association with a hepatic lesion, ${ }^{8}$ and then in a case of peritonitis. ${ }^{9}$
Reports of microsporidian ocular infection in patients with AIDS first appeared early in $1990{ }^{10}{ }^{11}$ Ocular infection presents with symptoms of foreign-body sensation, blurred vision or photophobia. Ophthalmological examination discloses conjunctivitis, decreased visual acuity, and a diffuse punctate keratopathy. Corneal or conjunctival scrapings or biopsies stained with Giemsa reveal oval dark-staining spores. Confirmation of the identity of the infecting microsporidia in these cases of hepatitis, peritonitis, and kerato-conjunctivitis has been by demonstrating their morphological similarity to Encephalitozoon cuniculi by electron microscopy. However, Didier et al recently isolated such microsporidia in cell culture from AIDS patients with kerato-conjunctivitis. Three such isolates were shown to be a new species, Encephalitozoon hellem, morphologically similar to Encephalitozoon cuniculi by electron microscopy, but distinct by SDS-PAGE analysis. ${ }^{12}$

\section{Case report}

A 26 year old married bisexual man was first shown to be HIV antibody positive at routine screening in 1986. In January 1988 he remained well with CD4 $0.40 \times 10^{9} / 1$, HIV p24 Ag + ve, $\beta_{2}$-microglobulin $4.1 \mathrm{mg} / \mathrm{l}$. He first developed bilateral conjunctivitis in October 1988. Nasal obstruction and discharge became prominent in February 1989. By this stage he was unwell with weight loss and relapsing fevers and investigations showed CD4 $0.19 \times 10^{9} / 1$, HIV p24 Ag $<100$ $>500 \mu / \mathrm{ml}, \quad \beta_{2}$-microglobulin $4.6 \mathrm{mg} / \mathrm{l}$ Zidovudine was commenced at $1 \mathrm{~g} /$ day with a good initial response. However, he continued to have episodic conjunctivitis, chronic nasal obstruction and discharge, as well as clinical and radiological evidence of sinusitis. This condition failed to respond to multiple courses of antibiotics and nasal decongestants. In June 1990 a diffuse punctate keratopathy was noted in both eyes, ENT examination showed multiple nasal polyps, and CT showed extensive opacities in the maxillary antra, and ethmoid and sphenoid sinuses, as well as minor cerebral atrophy. A full description of the ophthalmological findings and subsequent ocular response to treatment is being published elsewhere. ${ }^{13} \mathrm{He}$ was admitted to hospital in October 1990 with Pneumocystis carinii pneumonia and treated with intravenous pentamidine. Following recovery a formal nasal polypectomy was performed under general anaesthesia. After formalin fixation nasal polypectomy specimens

\section{Address correspondence to: Dr C Lacey \\ Accepted for publication 13 February 1992 \\ Department of}




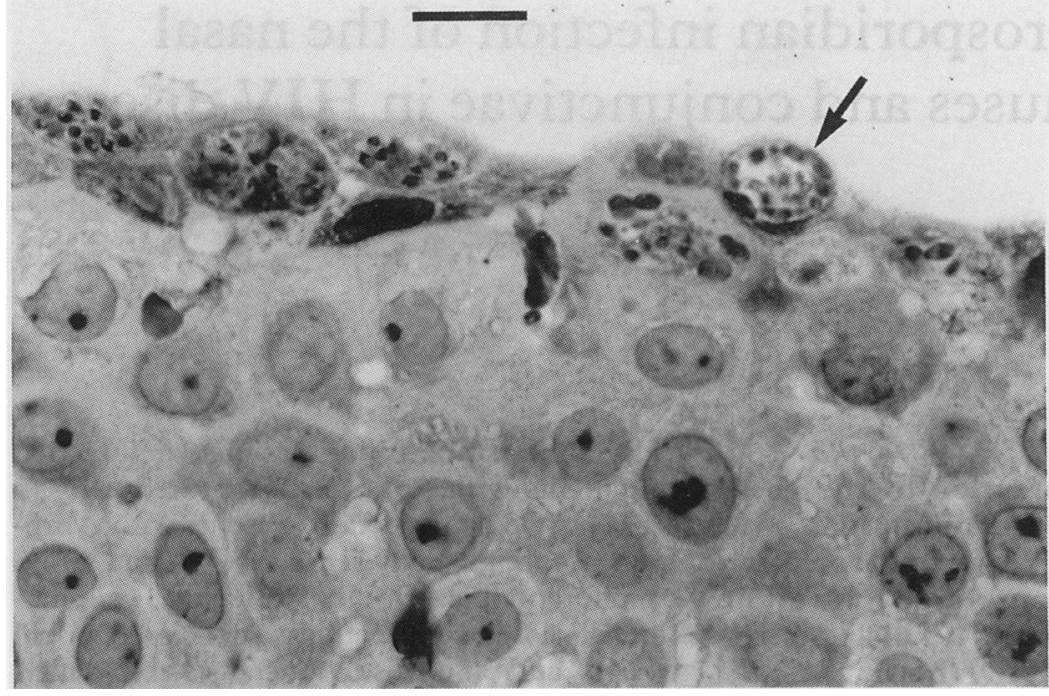

Figure 1 Nasal epithelium showing surface epithelial cells with cytoplasmic vacuoles containing multiple spores (arrowed). Scale bar represents $10 \mu \mathrm{m}$.

were embedded in paraffin wax (Ralwax 1, $\mathrm{BDH})$ and $4 \mu \mathrm{m}$ sections were stained with haematoxylin and eosin, PAS and Grocott stains. A specimen was also embedded in methacrylate derived plastic (Immunobed, Park Scientific Ltd, Northampton) and $2 \mu \mathrm{m}$ sections were stained by a two-stage MayGrunwald-Giemsa method. These preparations were examined by light microscopy. They revealed a polypoid nasal mucosa with a neutrophil infiltrate within the epithelium and a neutrophil, lymphocyte and plasma cell infiltrate in the adjacent submucosa. Many superficial epithelial cells contained numerous round

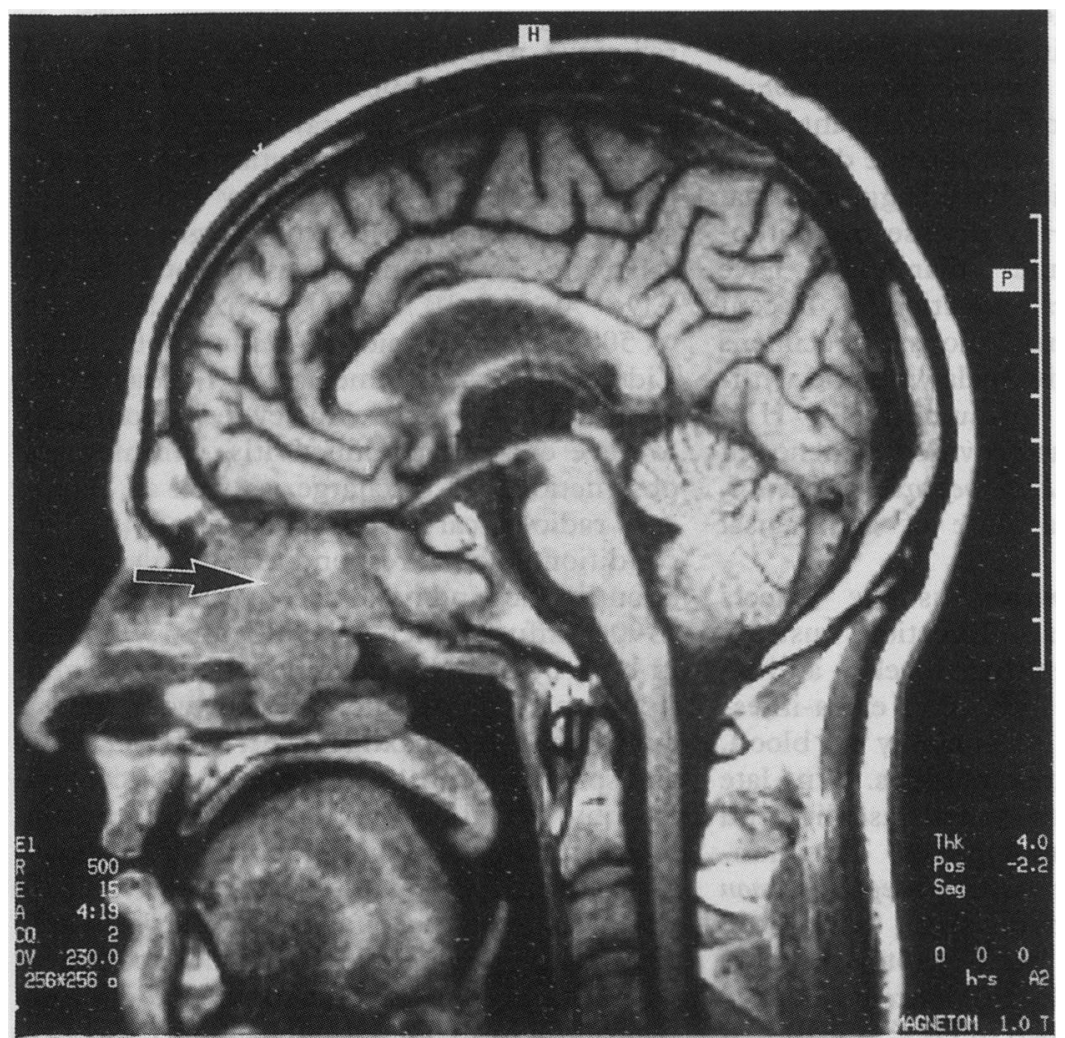

Figure 2 Sagittal magnetic resonance image showing inflammatory mass filling the nasal airway (arrowed) and sphenoid sinus. to oval organisms within the cytoplasm demonstrated by Giemsa staining (fig 1). These organisms measured approximately $1 \mu \mathrm{m}$ in diameter, were gram positive and did not stain with either PAS or Grocott stains. These light microscopic appearances were those of a protozoan infection of the nasal mucosa.

Corneal and nasal specimens were als examined by electron microscopy. Th⿱ showed a species of encephalitozoon infectine both epithelia. A full description of the ultri structure is being published separately. ${ }^{14}$

In January 1991 the patient developed AID dementia complex with spastic paraparesis of the legs and memory loss. Repeat CT and MR showed dilatation of third and lateral veif tricles, generalised involutional change an high signal material filling the nasal airway (fo 2).

By May 1991 nasal obstruction and disa charge were again problematic with multipto nasal polyps present on examination. Furth polypectomy was therefore performed. In vie of the observation of some degree of response $\theta$ f intestinal microsporidiosis to oral albendazolẹ (Blanshard C, personal communication therapy with this agent was instituted. Alberodazole $\mathbf{4 0 0} \mathrm{mg}$ bd was given for one month and obliquely oriented coronal CT was performed to demonstrate the nasal airway and sinus pre- (fig 3) and post- (fig 4) medical therapy: Both scans revealed erosion of the medial waly of the maxillary antra. During the course of treatment the patient's nasal symptomes improved, and there was significant regressio of sinus opacification. The patient remaine free of nasal symptoms until his death October 1991 from AIDS dementia complex

\section{Discussion}

The recent demonstration that three microsporidial isolates from cases of kerato-conjun? tivitis similar to our patient are distinct frong, but closely related to Encephalitozoon cunicut underlies our limited knowledge concernirg the epidemiology of microsporidiosis if humans. Bergquist and colleagues, for

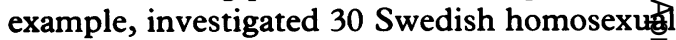

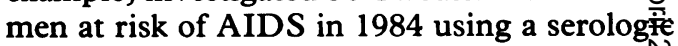
assay for antibodies to Encephalitozoon cunicuti and found a $33 \%$ antibody prevalence. ${ }^{15}$ Didier showed that antibodies against $E$ hellem cag cross react with $E$ cuniculi ${ }^{5}$ while any antibody response against Enterocytozoon bienusi has ngt been defined.

We have therefore described a man wien HIV infection and immune deficiency who has an opportunistic infection of the cornea, cof junctiva and nasal mucosae with a micro sporidian parasite similar to Encephalitozo® cuniculi and Encephalitozoon hellem. He has als radiological, computed tomographic and magnetic resonance imaging evidence \&f opacification of the maxillary antra, ethmow and sphenoid sinuses unresponsive to conver tional antibacterial therapy or nasal polypectomy, presumably caused by infection with the same organism. Late in the course of his microsporidian infection there was clear com- 


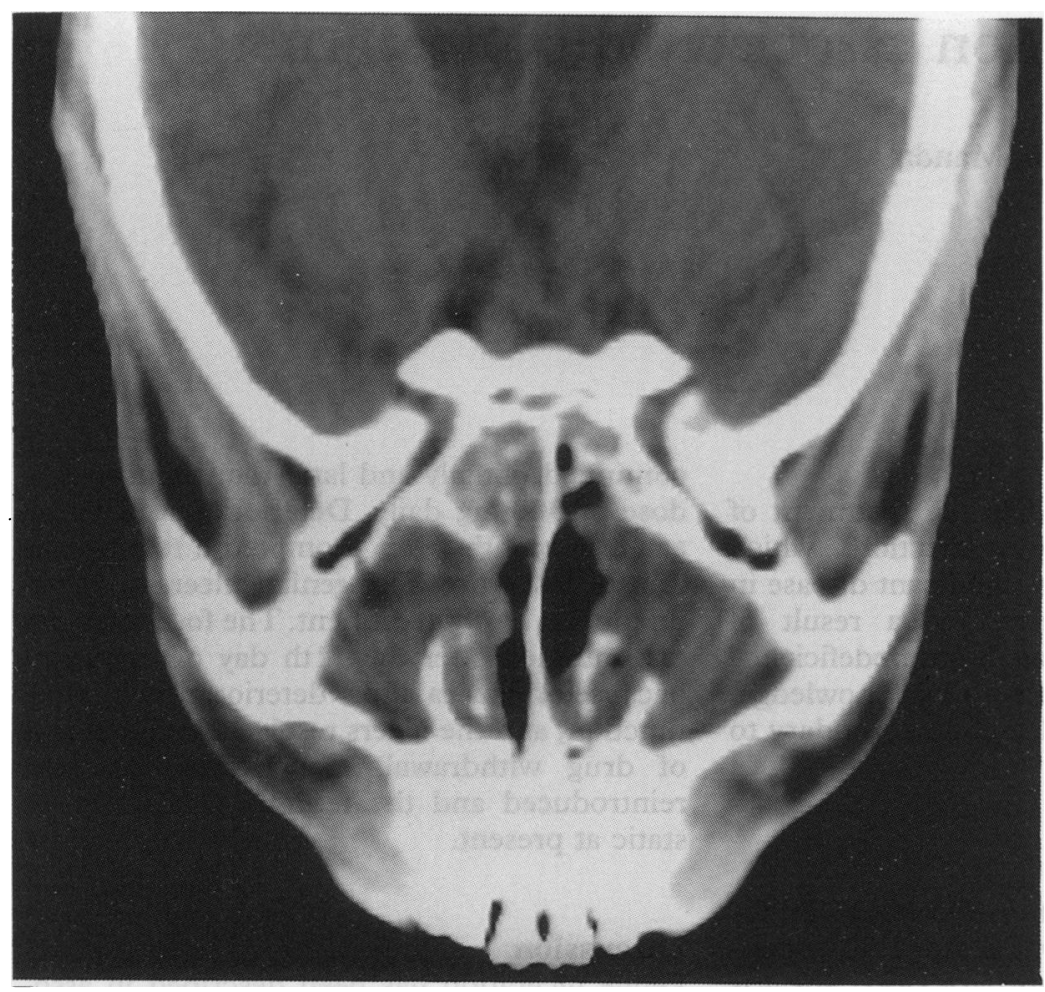

Figure 3 Oblique coronal CT post-second polypectomy pre-albendazole showing opacification of the maxillary antra and ethmoid sinuses.

puted tomographic evidence of bony destruction of the maxillary antra. Administration of albendazole produced symptomatic improvement and radiological regression of sinus infection. The manifestations of his ocular pathology are typical of previously described cases but this is the first case in an HIV infected subject described outside the USA. This subject has frequent contact with cats and dogs in his home environment and has never

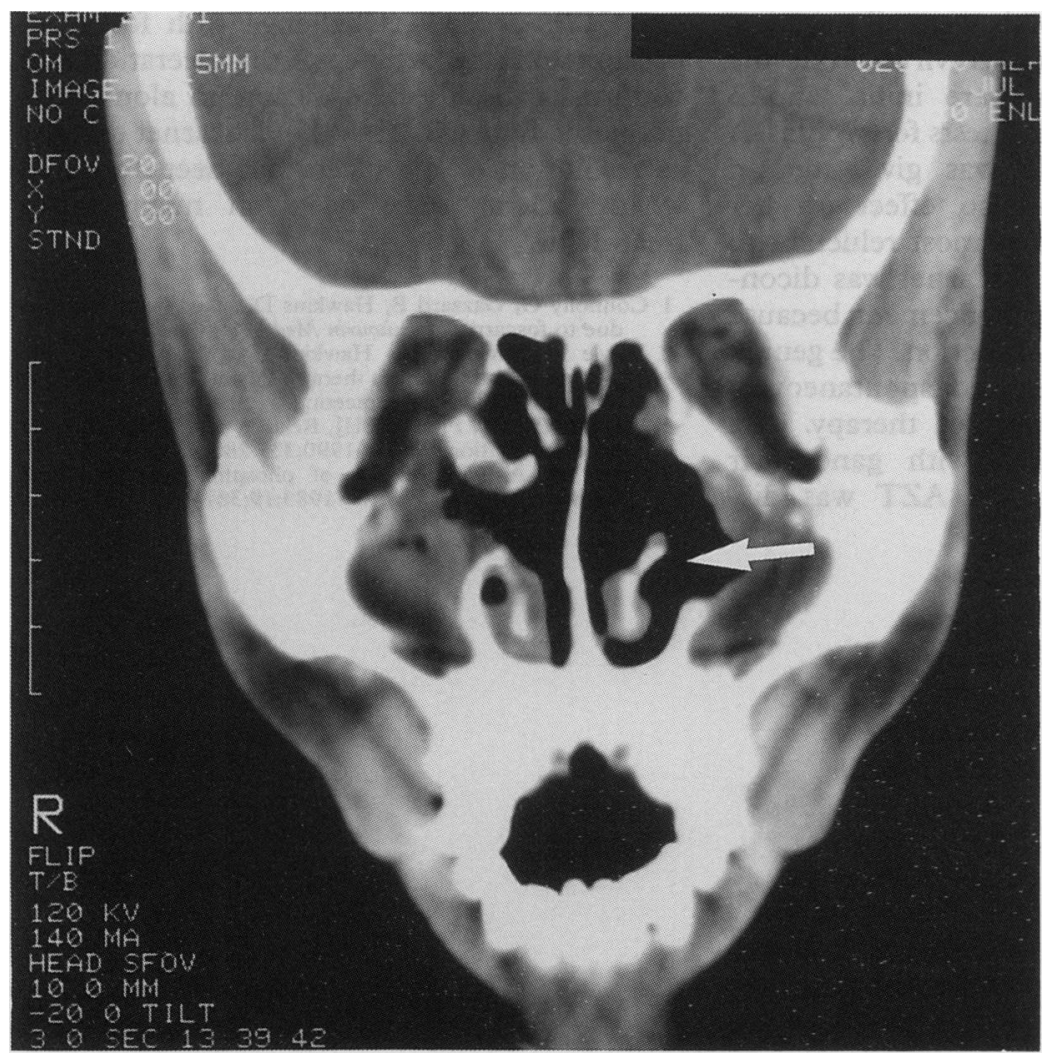

Figure 4 Oblique coronal CT post albendazole showing regression of opacification and deficiency of the medial wall of the left maxillary antrum (arrowed). travelled outside the UK. We know that Encephalitozoon cuniculi can spread horizontally among mammals via contaminated excreta ${ }^{16}$ and one could postulate domestic animals as a source of this patient's infection.

This subject's symptoms of ocular and nasal infection were first noted up to 2 years prior to demonstration of his microsporidial infection. This symptom complex developed relatively early in the course of his immune deficiency, in parallel with symptoms of systemic HIV disease at a time when his CD4 count was of the order of $0.200 \times 10^{9} / 1$. We found encephalitozoon easy to demonstrate by Giemsa stains or electron microscopy once suitable tissue specimens were obtained.

This case report extends the known tissue tropism of these organisms. This case appears to be a rarity, but precise definition of the frequency of microsporidial upper respiratory tract infection may depend on the development of serological assays. This in turn may depend upon propagation of such organisms and currently protozoa derived from the patient's polypectomy specimens are growing in tissue culture (Canning E U, personal communication). This man's infection appeared to respond well to albendazole. Encephalitozoon may be overlooked using light microscopy with certain stains and electron microscopy is at present vital in identification.

The authors thank Dr J T Lamb and Mr J D Fenwick for advice and assistance, Clare Midgley for secretarial services, and Stephen Toms for help with the illustrations.

1 Bryan RT. Microsporidia. In: Mandell GL, Douglas RG, Bennett, JE eds. Principles and Practice of Infectious Diseases. 3rd ed. New York: Churchill Livingstone, 1990:2130-4.

2 Modigliani R, Bories C, Le Carpentier Y, et al. Diarrhoea and malabsorption in acquired immune deficiency syndrome: A study of four cases with special emphasis on opportunistic protozoan infestations. Gut 1985;26: 179-87.

3 Dobbins WO III, Weinstein WM. Electron microscopy of the intestine and rectum in acquired immunodeficiency syndrome. Gastroenterology 1985;88:738-59.

4 Orenstein JM, Chiang J, Steinberg W, Smith PD, Rotter dam $\mathrm{H}$, Kotler DP. Intestinal microsporidiosis as a cause of diarrhoea in Human Immunodeficiency Virus-infected patients: a report of 20 cases. Hum Pathol 1990;21: 475-81.

5 Van Gool T, Hollister WS, Schattenkerk JE, et al. Diagnosis of Enterocytozoon bienusi microsporidiosis in AIDS patients by recovery of spores from faeces. Lancet patients by reco

6 Schattenkerk JKME, Van Gool T, Van Ketel RJ, et al. Clinical significance of small-intestinal microsporidosis in HIV-1 infected individuals. Lancet 1991;337:895-8.

7 Canning EU, Lom J. The Microsporidia of Vertebrates. London: Academic Press; 1986:208.

8 Terada S, Reddy R, Jeffers LJ, et al. Microsporidian hepatiti in the acquired immunodeficiency syndrome. Ann Intern Med 1987;107:61-2.

9 Zender HO, Arrigoni E, Eckert J, Kapanci Y. A case of Encephalitozoon cuniculi peritonitis in a patient with AIDS. Am J Clin Pathol 1989;92:3526.

10 Lowder CY, Meisler DM, McMahon JT, Longworth DL, Rutherford I. Microsporidia infection of the cornea in an Rutherford I. Microsporidia infection of the cornea in

11 Anon. Microsporidian kerato-conjunctivitis in patients with AIDS. MMWR 1990;39(11):188-9.

12 Didier ES, Didier PJ, Friedberg DN, et al. Isolation \& characterization of a new human microsporidian, Encephalitozoon hellem (n.sp) from three AIDS patients with kerato-conjunctivitis. J Infect Dis 1991;163:617-21.

13 Metcalfe T, Doran R, Rowland P, Curry A, Lacey CJN. Microsporidial kerato-conjunctivitis in a British patien with AIDS. Br J Ophthalmol 1992 (in press).

14 Canning EU, Curry A, Lacey CJN, Fenwick JD. Ultrastructure of encephalitozoon sp. infecting the conjunctival, corneal and nasal epithelia of a patient with AIDS. Eur $J$ Protistology 1992 (in press).

15 Bergquist R, Morfeldt-Mansson L, Pehrson PO, et al. Antibody against Encephalitozoon cuniculi in Swedish Antibody against Encephalitozoon cuniculi in Swedist

16 Cox JC, Gallichio HA, Walden HB. Application of immunofluorescence to the establishment of an Encephalitozoon cuniculi-free rabbit colony. Lab Anim Sci 1977;27:204-9. 